\title{
The Challenges of Migration from 2D to 3D Internet (3DI)
}

\author{
Agbaje, M.O, Awodele, O., and Joshua, J.V \\ Babcock University, Illisan- Remo, Ogun State, Nigeria
}

\author{
agbajeolugbenga@gmail.com; delealways@yahoo.com; \\ jvi@yahoo.co.uk
}

\begin{abstract}
The Internet world as we know it today has undergone far-reaching changes since its early days while becoming a critical communications underpinning our economic performance and social welfare. The 3D Internet is poised to replace the 2D Internet as we know it today. This paper takes a look at the two types of Internet, advantages and disadvantages and the future of the present Internet as we know it.
\end{abstract}

Key words: Internet, 2D,3D, communication, www

\section{Introduction}

The 3D Internet (3DI) is the set of 3D virtual and mixed reality worlds in the Internet. Here, users can experience, use and share with others for various applications (Kapahnke, Liedtke, Nesbigall, Warwas, \& Klusch, 2010) .The Internet from onset is a 2D Internet because most of the graphical user interface (GUI) at this stage are two-dimensional. The 3D Internet use of 3D graphical user interface (GUI) which makes it is a powerful new way for reaching consumers, business customers, co-workers, partners, and students, and is inherently interactive and engaging. Virtual worlds provide great 3D experiences that mirror real life. Almost anything in the real world can be reproduced in the 3D Internet with the added benefit because someone can experience it from the comfort of their home or office.. The paper takes a look at the 3D Internet and the challenges of moving from the Internet as we know it to the future Internet, that is, 3D Internet.

The 3D technology has grown into Internet. This was one of the main trends in the large international technology meeting in CEBIT (CEntrum der Buro und Information Technik). CEBIT is the world's largest and most international computer expo. Earlier Internet has lacked a common standard for 3D on Internet. Now several large Internet companies have agreed on a new open 3D Internet standard.

The work started in late 2009, and the companies could agree on the 3D-standard WebGl. This

Material published as part of this publication, either on-line or in print, is copyrighted by the Informing Science Institute. Permission to make digital or paper copy of part or all of these works for personal or classroom use is granted without fee provided that the copies are not made or distributed for profit or commercial advantage AND that copies 1) bear this notice in full and 2) give the full citation on the first page. It is permissible to abstract these works so long as credit is given. To copy in all other cases or to republish or to post on a server or to redistribute to lists requires specific permission and payment of a fee. Contact Publisher@InformingScience.org to request redistribution permission. standard is based on the open standard OpenGL. HTML5 is the newest version of the Internet programming language HTML, opens for the new 3D standard WebGl. This enables users of Internet to see 3D on Internet without plug-ins.

The Internet's roots are in the U.S. during the late 1960s. The Web was invented 20 years later by Sir Tim BernersLee. The Internet world has undergone far-reaching changes since its early days 
while becoming a major communications supporting our economic performance and social welfare. Internet currently has more than one billion users worldwide and is poised to spread its infrastructure providing anywhere, anytime connectivity. With the further use of wireless technologies, the number of users of the Internet is expected to jump to some 4 billion few years ("The future of Internet," 2008). The Internet is a massive network of networks. It connects millions of computers together globally in which any other computer can communicate with any other computer as long as both connects to Internet. The web connects people. Information that travels over the Internet does so by various languages know as protocols. It does not use web browsers. The Web, running on the mostly invisible Internet, is what you see and click on in your computer's browser. Worldwide Web (WWW) is a way of accessing information over the Internet. It is an information sharing model that is built on the top of Internet. The web uses hypertext transfer protocol (HTTP), to transmit data. It is also utilizes browsers such as Internet explorer or Firefox to access web documents called web pages that are linked by hyperlinked.

The WWW started as a document repository and it has rapidly transforming to a full-fledged virtual environment that promotes services and communication. The Semantic Web and Web 2.0 movements are intermediate steps of a natural evolution towards a new paradigm: the 3D Internet. The idea seems incremental in the sense that it adds 3D graphics to the current Web. It is revolutionary for it provides virtual environment that promotes services, interaction, and communication. From this perspective, the 3D Internet is seen as the evolutionary end point of continuing efforts such as Web 2.0 and Semantic Web (Alpcan, Bauckhage, \& Kotsovinos, 2008).

The 3D Internet promises to bring real life feeling to our interaction on the web. But for most of the users now, the Internet is a familiar, comfortable medium where they can communicate with one another, get our news, shop, pay our bills, and more. But navigating hierarchical data structures is often cumbersome for large data sets. Unfortunately, there is a better alternative way of organizing data which everybody knows and uses. We spend all our lives in a 3D world navigating between places and organizing objects spatially. We rarely need search engines to find what we are looking for and our brains are naturally adept at remembering spatial relationships.

It is predicted that, within 7-10 years, the dominant Internet interface is likely to be the 3D Metaverse. This is next-generation and high-definition media-rich Web 3D. Metaverse will be gradually absorbed, and integrate with today's flat Worldwide Web and its early 3D applications like Google Earth and Second Life .The European Commission is even officially recognizing the 3D Internet as a strategic research direction, as seen, for example, in their Seventh Framework Programme (FP7) call for proposals issued on 19 November 2008.

\section{Literature Review}

Here we review what has led to developing the 3D Internet and the various research groups and companies involved in developing the technology. Figure 1 shows some of the companies involved in 3D Internet. 


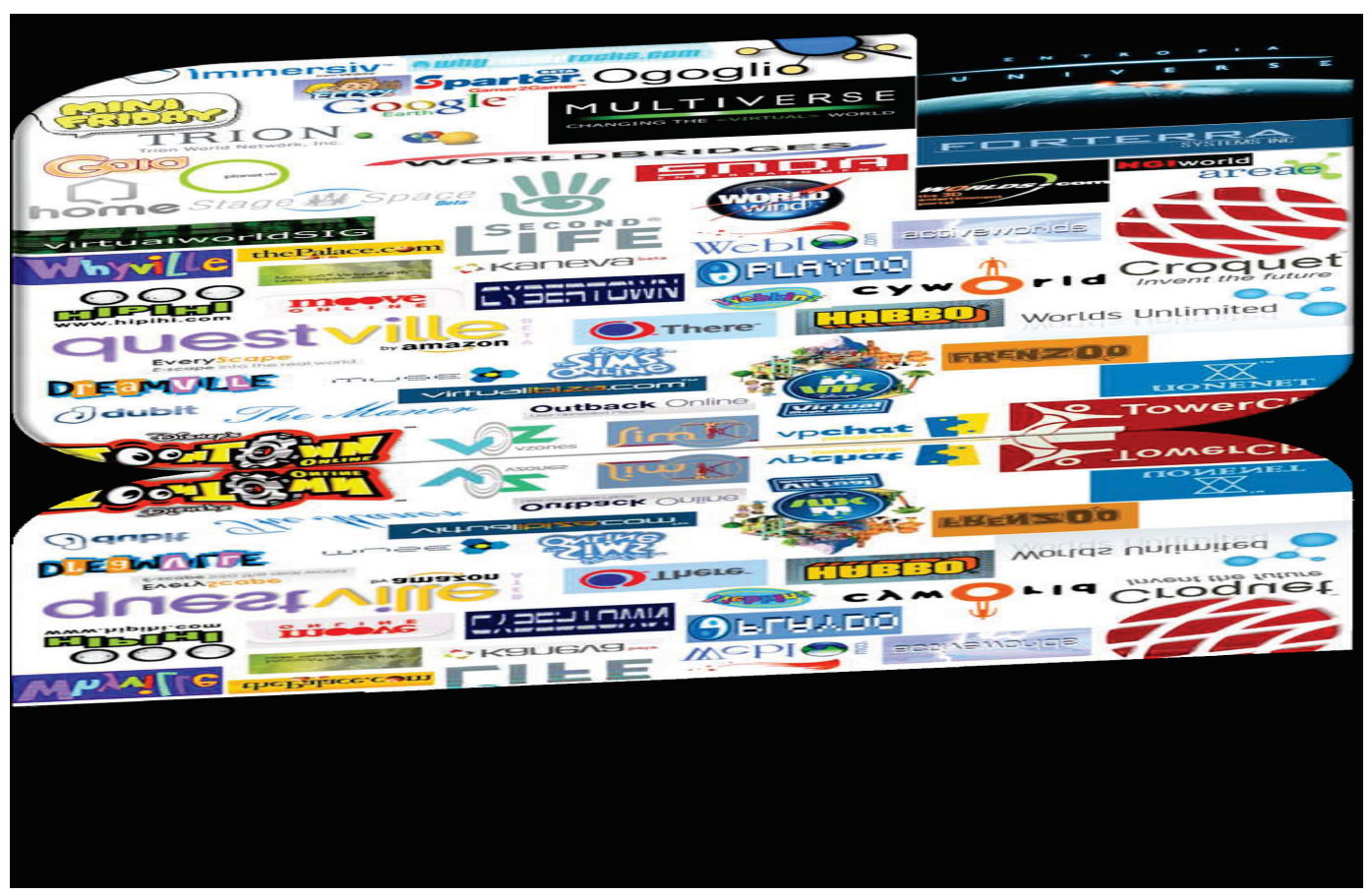

Figure1. Snapshots of 3D Internet today (Rattner, n.d.)

Some of the companies involved include questville, Trion, SecondLife, GoogleEarth, Ogoglio, Sparter, cyworld, Multiverse and so on.

In 1982, the Internet protocol suite TCP/IP (Transmission Control protocol/Internet Protocol) was standardised and the concept of world-wide network of fully interconnected TCP/IP networks called the Internet was introduced. All based on research agency ARPANET (Advanced Research Project Agency Network) of the US Department of Defence. The TCP is a Connection-oriented transport protocol of the Internet architecture a provides a reliable, byte-stream delivery service while the IP also known as IPv4 is a protocol that provides a connectionless, best-effort delivery service of datagrams across the Internet. The packet switching was based on the concept and design of Paul Baran, Donal Davies and Lawrence Roberts of the Lincoln Laboratory. The TCP/IP set of protocols were devoloped for ARPANET by computer Scientist Robert Kahn and Vinton Cerf (wiki).

W3C consortium created the protocol HTTP (Hyper Text Transfer Protocol). It is used to communicate between Web browsers and Web servers. It is an application-level protocol based on a request and reply model and used in the Worldwide Web. HTTP uses TCP connections to transfer data.

The Internet (or Internetwork) is a collection of (possibly heterogeneous) packet-switching networks interconnected by routers while the Internet is the global Internet based on the Internet (TCP/IP) architecture, connecting millions of hosts worldwide.

The first Web browser was developed in 1990. It was called World Wide Web and later Nexus. In 1993 Mark Andresen created and release Mosaic later called Netscape. This was the world first popular browser which made the WWW system easy to use and more accessible to average person. They are the major milestone in the history of the web. In web development, the web1.0 a set of static web pages while the web2.0 is the phrase used after the dot.com crash. It is more interactive in nature with introducing social networking and interactive multimedia. After web2.0 we have the semantic web, which is an extension of WWW that enables people to share content be- 
yond the boundaries of application and websites. It proposes to help computer "read" and use the web. This idea is carried out by adding metadata to existing web pages to make it to machinereadable form. It also uses agent functioning.

\section{D Internet History}

In 1995 the Virtual Reality Markup Language (VRML) was developed. The interest for 3D was small; all focus was toward general Internet HTML. VRML (Virtual Reality Modeling Language) was development of the Virtual Reality Markup Language from before 1995.

VRML was a standard file format for representing 3D Internet/ web. Interactive vector graphics could now be designed with the World Wide Web in mind. The VRML allowed 3D vertices and edges for 3D Internet to be show with colours. Different textures and transparency could be added. This allowed the web browser to click on graphical components. This opened for animation, sounds, lighting and other interesting features. The WRML files (often called "worlds"), has the extension *.wrl. The VRML has been superseded by the standard X3D, formed by the Web3D consortium. The X3D was accepted as international standard by ISO.

The first VRML version was ready in November 1994. The complete version is VRML97, which was the latest version of the language. VRML was newer much used for 3D on Internet. The reason for this may have been the lack of available bandwidth for users at this time. In the 2000s, many tried to improve the quality level of virtual effects in VRML. We have also seen towards the level of DirectX 9.0c. But this was using proprietary solutions.

The VRML has been superseded by the standard X3D, formed by the Web3D consortium. The X3D was accepted as international standard by ISO. We have a new 3D on Internet standard. $\mathrm{X} 3 \mathrm{D}$ is the file format for 3D computer graphics. The X3D has the extension *.x3d, *.x3db, *.x3dv. The X3D support multi stage / texture render. It supports shader with lightmap and normal map. Latest version of X3D allows realtime environment and reflection towards lighting. The X3D can also use content from other opens source standards like XML, DOM and XPath.

A X3D applet is software in the web browser which displays content in 3D/3D Internet. The OpenGL 3D technology is used to display 3D content within X3D. The X3D works with several different web browsers (Internet Explorer, Safari, Firefox, Opera..). It also can be used on different operating systems (MS Windows, Mac, OS X, and Linux. This gives us 3D Internet. The X3D is most likely to become the standard for 3D on Internet in the future, due to its integration with the new Internet language HTML5 and other XML version like Mathlab, SVG and so on.

\section{Development of Computer User Interface}

The 3D user interface - avatar - is the crucial difference between the web and the 3D Internet, an avatar is the graphical representation of the user or the user's alter ego or character. It may take either a three-dimensional form, as in games or virtual worlds. The word avatar originates in Hinduism, where it stands for the "descent" of a deity in a terrestrial form. The use of the term avatar for the on-screen representation of the user was coined in 1985 by Chip Morningstar and Joseph Romero in designing Lucas film's online role-playing game Habitat. Avatars in video games are essentially the player's physical representation in the game world. In most games, the player's representation is fixed, however increasingly games offer a basic character model, or template, and then allow customization of the physical features as the player sees fit. Avatars in non-gaming online worlds are two- or three-dimensional human or fantastic representations of a person's inworld self. Such representations are a tool which promotes exploring the virtual universe, or acts as a focal point in conversations with other users, and can be customized by the user. 
The user...

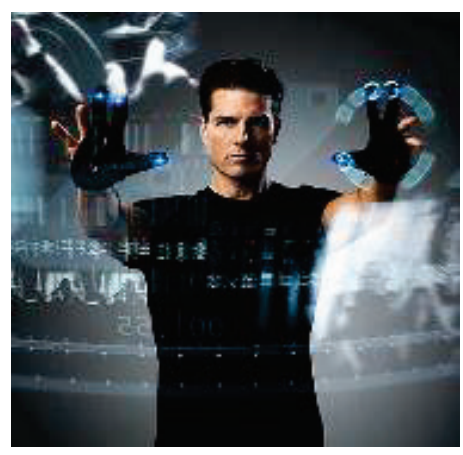

\section{...selects an avatar}
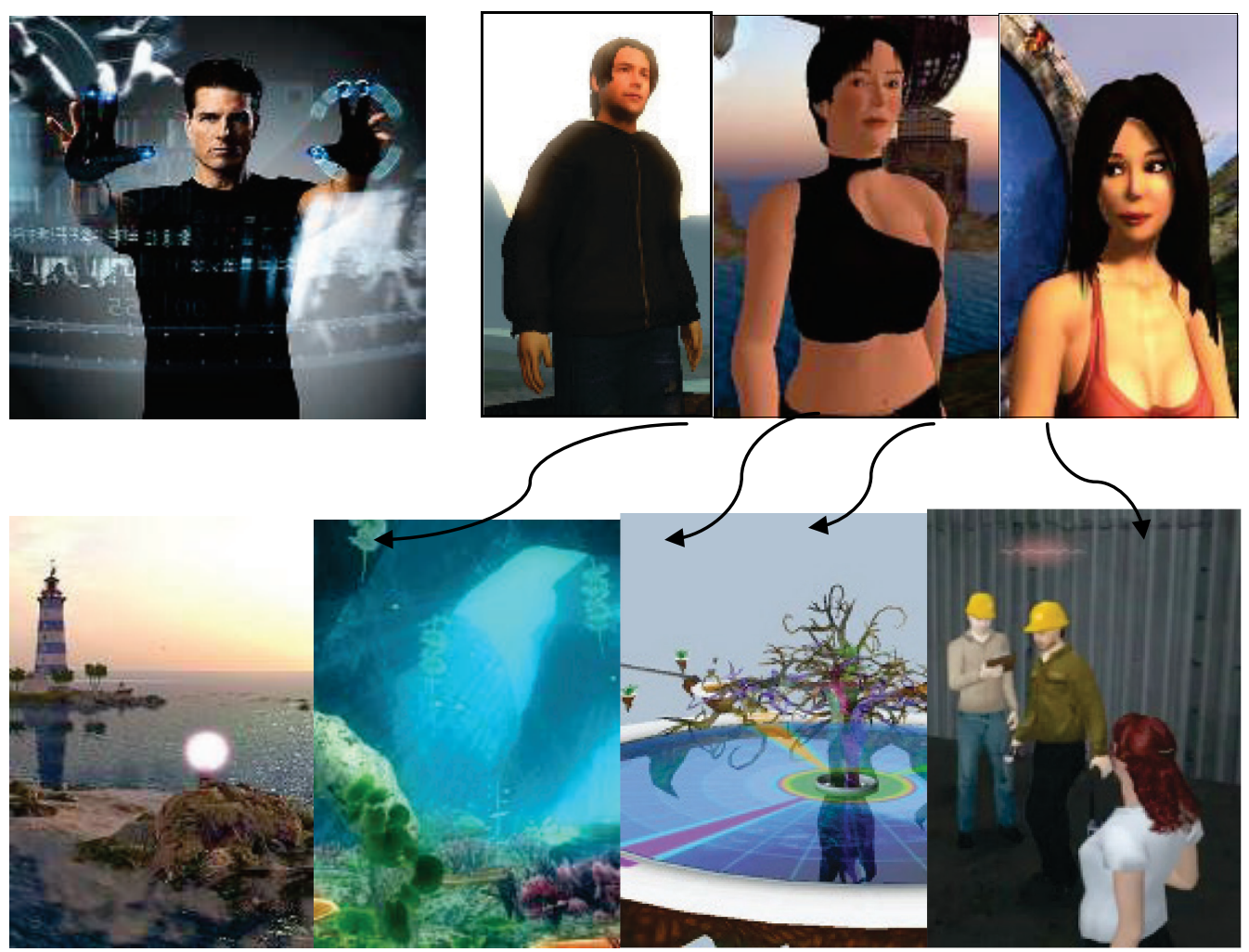

Figure 2: 3D world interface(Source: Pirkola, 2012)

The historical development of interface is summarized below:

\author{
Punch Cards • 1890, IBM \\ Text $\quad 1950$ \\ 2D graph $\quad \bullet 1973$, Xerox PARC・Desktop, WIMP \\ Networked \\ 2D graphics $\bullet 1990 \cdot$ WWW \\ Networked 3D graphics $\bullet 2013 \bullet$ 3D Internet
}

\title{
2D and 3D Internet
}

The 2D examples include today- popular retailer like: Drugstore.com, Macdonalds.com and Walmart.com. The 3D examples include the following: Google earth, Multiverse, and Second life.

According to a Cisco (Nasdaq: CISCO) blog, it says that while the transition to 3D Internet is in its early stages, it's inevitable. He said 3D Internet can be thought of as a set of interconnected virtual worlds that users can visit to use services, 'teleporting'(move somewhere without travel- 
ling) from one world to another. It uses many of the same basic technology components as the 2D Internet- a browser, search engine and servers.

3D Internet makes virtual communities and collaboration - being and doing together - possible. 3D Internet is the next wave after the current 2D web. 3D Internet consists of interconnected services, presented as virtual worlds. Virtual worlds are 3D environments user enters with his avatar. Virtual worlds may be considered as advanced web sites and avatars as user profiles. The difference lays in collaborative content creating where user is part of building his virtual experience with other users' avatars and service provider.

The 3D Internet uses 3D graphics and, sometimes, avatars. That's what will make the new Internet more social than you know today. While 3D graphics are already seen in online virtual worlds such as Second Life, the 3D Internet won't be limited to recreation (Ohnesorge, 2013). What makes these new worlds possible? Several technology platforms exist. In 2007, the realXtend project kicked off in Oulu, HiTech hub in Northern Finland. The realXtend project aimed to develop the de facto -standard for 3D Internet. Currently, realXtend developed globally as an open source project, lead from Oulu by a non-profit organization. The realXtend people believe that open source makes the best technology available for all, and the true value of the 3D Internet does not lie in the platform but in the content.

Pirkola (2012) gives the differences between 2D and 3D Internet below:

\section{D Internet}

User experience:

Comparable to phonebook

Back button is the most commonly used key

Solitary

Emotion,non verbal, communication difficult

To express

Depicting oneself,blog,homepage

Isolates users

Powered by search engines, depends heavily on

Key words

\section{D Internet}

User experience:

Life -like

Intuitive

Interactive,Strong cohesion

Emotion,non-verbal communication easy to express using avatar Depicting onself: avatar, virtual world social interaction inherent Information linked to avatar and spaces not key words.

\section{Virtual Reality and Virtual Worlds}

Virtual reality and Virtual World are major distinctions that separate a 2D Internet from 3D Internet. Virtual reality is a technique by which a computer simulates a three-dimensional physical environment using visual and auditory stimuli within which people can interact to affect what happens in the simulation.

A virtual world is an online community the takes the form of a computer-based simulated environment through interact with each other, use and create objects. Virtual worlds have off-line, real world components and applications. The application domains include tourism, social, medical, commercial, e-commerce entertainment, games, and education.

Sivan (2008) of the Metaverse1 Project Consortium (http://www.metaverse1.org ) defines three dimensional (3D) virtual worlds as a '3D3C' combination of:

1. 3D: controllable graphical environment (with 3D spatial audio) - one can zoom, change camera position, etc.; 
2. Community: real people can create groups, helped by a system that allows communities to blossom;

3. Creation (or 'plasticity'): there are ways to build and edit content, services, and various stuff; and

4. Commerce: the ability to attach real economic value (real money) to services, as necessary (e-commerce and fund raising activities on the flat, conventional Web).

Between 2006 and 2008 the medical and public health communities around the world have shown a growing interest in using 3D virtual worlds like Second Life for health education, community outreach, and training and simulations purposes. Second Life and Twinity can be approached as 3D social networks, where people can also collaboratively create and edit various objects in the virtual world in real time, besides meeting each other and interacting with existing objects (Kamel Boulos, Hetherington, \&Wheeler, 2007). These worlds are seen to as the forerunners of Web 3D, the next major iteration of the Internet (also sometimes called the Metaverse) that will follow in the coming years (2015-2020).

The popular 'Second Life in Education' and references list dozens of uses of 3D virtual worlds. They include: Distance and flexible education, Presentations, panels and discussions, Training and skills development, including self-paced tutorials. Treasure hunts and searches, Role-plays and simulations, Data visualisations and modelling are supported. Support and opportunities for people with disabilities (stroke, autism, etc.) are also part of the uses. Real estate practice (e.g., visit accurate property replicas in the virtual world), Product design, user-testing and market research; and Urban or city and healthcare planning and prototyping can be performed (Kamel Boulos, Ramloll, Jones, \& Toth-Cohen, 2008).

\section{Second Life (SL)}

SL is a three-dimensional, virtual world where online residents use a three-dimensional character (or "avatar") to navigate their interaction with other online residents (Mennecke, Triplett, Hassall, Conde, \& Heer, 2011). Residents can customize their avatar and configure it according to their wishes, ranging from different humanoid (i.e. woman-like, man-like, childlike), para-humanoid (i.e. vampires, zombies, etc.), and non-humanoid like figures (i.e. cars, animals, robots, etc.). Residents can use these avatars to interact with other residents, and take part in karaoke, dancing, role-playing, and sexual intercourse. Depending on a person's personality preferences, one can choose the online interaction level they wish to engage in when engaging in virtual worlds such as SL. Ranging from solitary activities such as building a home, to more interactive activities such as role-playing in simulations with many others, residents can personalize their chosen activity according to their personal inclination and personality type preferences.

\section{Architecture of 3D Internet}

The principles the 3D Internet shares with its predecessor include open and flexible architecture, open protocols, simplicity at the network core, intelligence at the edges, and distributed implementation. User surfs the 3D Internet and uses teleports to move between independent worlds or services.(Tansu et al,2008 ).Terms adopted by Tansu et al with the 3D Internet are Universe, World, Webplace against the 2D Internet we have WWW, Website and Subdomain. Figure 3a and $3 \mathrm{~b}$ shows $3 \mathrm{D}$ Internet architecture. 


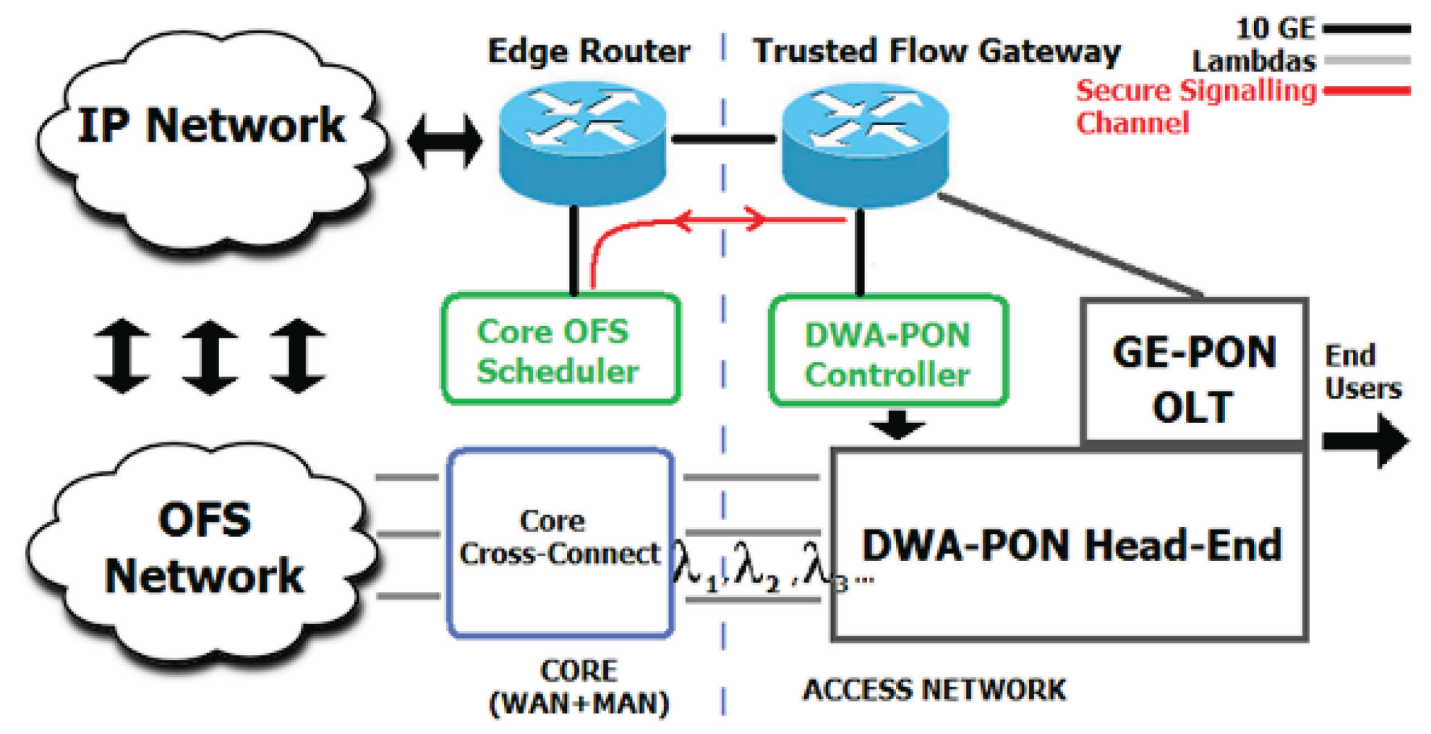

Figure 3a: 3D Internet architecture.

Figure 3a shows network components. It is divided into two parts . The Core (WAN and MAN) and the Access Network. WAN is Wide Area Network and MAN is Metropolitan Area Network. A node connected to two or more networks is called a router or gateway. Router connects any number of LANs and .Uses standardized protocols to move packets efficiently to their destination. It works much as a switch for sending messages from one network to another. The OLT represent the Optical Online Transmission to allow for computer connections. The 10 GE-EPON (Gigabyte Ethernet passive Optical Network) allow computer network connects over telecommunication line. The Optical Connectivity Feedback Network(OFS) interacting with the Internet protocol Network(IP Network). The DWA- PON (Dynamic wavelength allocation Network-Passive optical Network) Controller is for faster access across network.

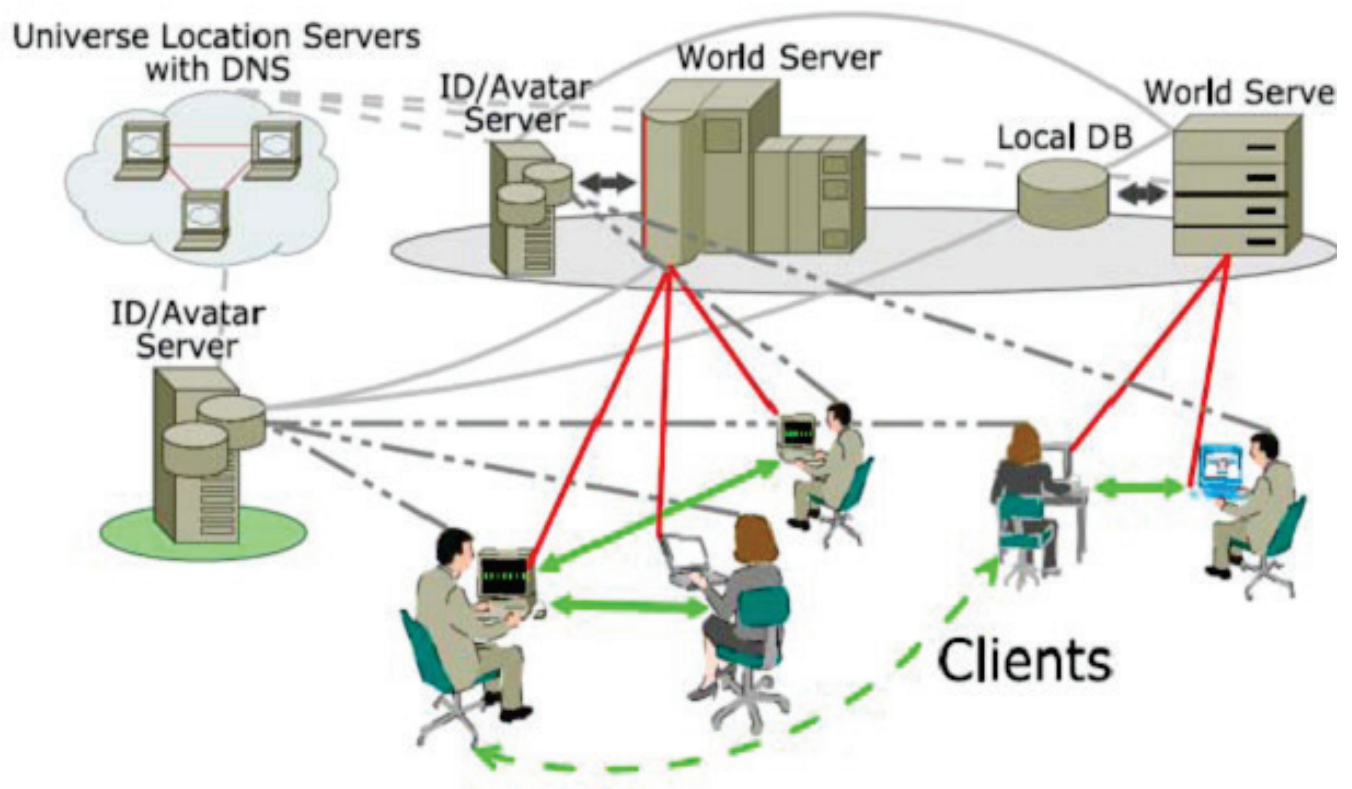

Figure 3b: 3D Internet Architecture (Alpcan et al., 2008) 
Figure 3b shows a proposed Internet architecture by Alpcan et al. (2008). The 3D Internet shares principles and underlying architecture of the current Internet as well as many semantic web concepts. The operational principles the 3D Internet shares with its predecessor include open and flexible architecture. Others are open protocols, simplicity at the network core, distributed implementation and intelligence at the edges. Decision making is not only at the centre but at the nodes to make room for multi application control network that is reliable.

The terms universe, world, and webplace are 3D counterparts of WWW, website, and sub domain, respectively. The component's functionality is described briefly below:

\section{World servers}

Provide user- or server-side created, static and dynamic content making up the specific webplace (3D environment) including visuals, physics engine, avatar data, media, and more to client programs. A world server has the important task of coordinating the co-existence of connected users, initiating communication between them, and ensuring in-world consistency in real time. They may also facilitate various services such as e-mail, instant messaging, and more.

\section{Avatar/ID servers}

Virtual identity management systems containing identity and avatar information as well as inventory of registered users and providing these to individual world servers and relevant client programs (owner, owner's friends) while ensuring privacy and security of stored information. Avatar/ID servers can be part of world servers.

\section{Universe location servers}

Virtual location management systems similar to and including current DNS providing virtual geographical information as well as connection to the Internet via methods similar to SLurl (Second Life url). They can also act as a distributed directory of the world, avatar servers and users.

\section{Clients}

Browser-like viewer programs running on user's computers with extensive networking, caching, and $3 \mathrm{D}$ rendering capabilities. Additional components of the 3D Internet include webplaces (replacing websites) and 3D object creation or editing software (i.e. easy-to-use 3D modelling and design programs such as Sketch-Up and standardised mark-up languages and communication protocols). Emergence of new software and tools as well as the ones mentioned should naturally be expected (Alpcan et al., 2008).

\section{D Internet Challenges}

1. Platform performance: FP intensive client/server, Constant bandwidth and Low latency. Therefore, the network has to be designed intelligently to overcome these challenges of low latency due to high graphics. The use of PC with 20X GPU and 3XCPU performance.

2. User created contents(UCC): Portability across worlds, Easy-to-use tools, Realistic rendering. This is online content that has been created by an Internet user. Tools must be provided to create this content. They must produce tools for content creation freely. Site like YouTube allow user to distribute their content in ways that were impossible 10 years ago. Some virtual site gives users chance to create individual oriented content and may limit the amount that can be environmentdirected. Each world would have a cost associated with each types of content. There are also different revenue models. 
There is no widely accepted definition of UCC, and measuring its social, cultural and economic impacts are in the early stages. Wunsch-Vincent and Vickery (2007) define UCC is defined as: i) content made publicly available over the Internet, ii) which reflects a certain amount of creative effort and iii) which is created outside professional routines and practices While measuring UCC is in its infancy, available data show that broadband users produce and share content at a high rate, and this is particularly high for younger age groups (e.g. 50\% of Korean Internet users report having a homepage and/or a blog). Given strong network effects a few platforms draw large amounts of traffic, and online video sites and social networking sites are developing to be the most popular websites worldwide (Wunsch-Vincent \& Vickery, 2007).

3. Simulation services: Dense avatar scaling, diverse client types, Unified graphics/physics. Simulation services can be done on server side or client side. Tools and modalities must be agreed on.

4. Ecosystems: Stimulation standards, 3D browser standards, Identity with anonymity. Capacity to deliver unified and intuitive user experience across multiple devices including HDTV, Blu-ray player, tablets and more.

5. The management of multiple identities. Identity management is prime so that while on several virtual world the individual has the same identity and can be identified. In this conception, conscious and aspect of self are increasingly externalized as distributed into both 2D and 3D digital personas(e.g Facebook,twitters,3D virtual world) reflecting any number of combination of now malleable traits of race, gender ,age, style, body type, personality and physical health.

6. Monetization of virtual assets. Each virtual world now has their currency system. Second life uses Linden Dollar. What is the role of this currency another world like Ogoglioverse and others? In the same way that web application like eBay and Amazon has their own payment system, services built on the ogoglio platform will have their own. The ogoglio based services won't be as tightly bound as the Second Life grid, so there isn't going to be a single company which can control a currency or exchange ("The future of the Internet," 2008; Zahariadis, Daras, \& LasoBallesteros, 2009).

7. Applicable rules or privacy of "digital avatars": One of the main values prominent in the report around the effects of technology on modern society, is privacy. Privacy is seen as a basic human right in all western democracies, and is frequently argued to be an essential precondition for establishing other human rights.

Many of them relate privacy to control: The individual's control over who has access to her personal life and information. Control over interactions with others, Control over handling one's personal information, against surveillance and observation, and a physical control over one's personal space. A main characteristic of Second Life is anonymity, which enables users to distinguish their on-line, in-world identities (their avatars) from their off-line identities. Many users state that they feel like their Second Life identity is not identical, and even significantly different from their "real life" identity. In this sense Second Life is different from social networks such as Facebook, which needs users to login and use their genuine names and identities.

8. Speed: Internet speed is one of the most significant implications that are being faced by the 3D Internet. Many countries in the world are in a state to fulfil the Internet speeds that are required for carrying out the $3 \mathrm{D}$ Internet. With their introduction of $3 \mathrm{G}, 4 \mathrm{G}$ etc. standard there is an increase in speed consideration.

$3 \mathrm{G}$ is the third generation of telecommunication standards and technology for mobile networking. $3 \mathrm{G}$ networks are wide-area cellular telephone networks that evolved to incorporate high-speed Internet access and video telephony. It is expected that $3 \mathrm{G}$ will provide higher transmission rates: a minimum speed of $2 \mathrm{Mbit} / \mathrm{s}$ and maximum of $14.4 \mathrm{Mbit} / \mathrm{s}$ for stationary users, and $348 \mathrm{Kbit} / \mathrm{s}$ in 
a moving vehicle. Hence, with introducing the $3 \mathrm{G}$ technology, the speed implications involved with the 3D Internet would be solved soon.

9. Hardware: The major hardware implication in the 3D Internet is the display. The use of 3D goggles is one solution that can is employed to overcome the problem. As we know there are various range of 3D goggles available in the market, we can select from these wide variety of 3D goggles. And the cost of these goggles is even less, so this prospect can be considered in the preliminary stages of the 3D Internet, later these could be upgraded with the latest technologies which could be used to implement/ display the 3D data.

Use of Vision Station as a monitor or display for the 3D Internet, Vision Station is a computer display technology developed by Elumens that provides 180 degrees of viewing angle for its users. Current computer screens have at most a 50-degree field of view and needs the user to move the controller in order to see the images that are not on the screen. This motion is unnatural because in the real world, users use their peripheral vision to see things beyond the direct line of sight. This new display technology will address this limitation of standard computer monitors.

\section{Visualisation and Interfaces}

A first approach to 3D Internet is the problem of visualisation and interfacing. Devices that can perform such a goal include PETs (Personal Enhanced Terminals, usually known as Mobile Phones) and PARTSs (Personal Augmented Reality Three-dimensional Spectacles). PETs are capable of creating a holographic image, therefore, enabling the visualisation of 3D images and videos. PARTSs make up an advanced version of today's multimedia glasses, making it possible to visualise 3D images and even mixing them with real images (Correia, 2009)

\section{Obstacles to Commercial Success in 3D Worlds}

Advertisers, marketers and organizations have yet to capitalize on the vast potential of the 3D Internet. Causes inhibiting the commercial usability of virtual worlds include:

i. The limited effectiveness of traditional media techniques such as fixed-location billboards when applied to virtual worlds. In the 3D Internet, participants have complete control over where they go and what they do - and can move their avatars instantly through virtual space. What is required is a means for making content readily available to people not only at specific points, but throughout virtual worlds.

ii. Lack of an effective way for enabling people in virtual worlds to encounter commercial content that heightens their virtual experience. Because participants have a choice in whether to interact with an offering, it is essential that it be viewed as relevant and valuable to their particular goals in the 3D Internet.

iii. An inconsistent means for enabling in-world participants to easily interact with and access video, rich multimedia, and Web content.

The lack of a cohesive means for advertisers and content providers to receive the detailed metrics required to measure success.

\section{Applications of 3D Internet}

\section{Education}

3D Internet can serve as a platform for education by many institutions, such as colleges, universities, libraries and government entities. There are subjects such as chemistry and English in which Instructors and researchers would favour 3D Internet because it is more personal than traditional distance learning. 


\section{Religion}

Religious organizations can make use of the 3D Internet to open virtual meeting places within specified locations.

\section{Embassies}

We could create embassies in 3D Internet, where visitors will be able to talk face-to-face with a computer-generated ambassador about visas, trade and other issues.

\section{Live Sport Entertainment}

Popular forms of live entertainment could also be placed into the 3D Internet. Many sports allow the users to watch or join many popular activities. Sporting leagues like Cricket, Football, Professional Wrestling, boxing, and auto racing could be placed in the 3D Internet for its users to play in the $3 \mathrm{D}$ environment.

\section{Arts}

The modelling in 3D Internet would allow the artists to create new forms of art, that in many ways are not possible in real life due to physical constraints or high associated costs. In 3D Internet artists could display their works to an audience across the world. This has created an entire artistic culture on its own where many residents who buy or build homes can shop for artwork to place there. Gallery openings even allow art patrons to "meet" and socialize with the artist responsible for the artwork and has even led to many real life sales. Live music performances could also be enabled in the 3D Internet.

\section{Conclusion}

The Internet revolution is taking place rapidly and there is a need to be part of it. The TV displays are also moving from 2D to 3D therefore in no distant time 3D Internet will be here. The gaming world has advanced and many training are already being done in $3 \mathrm{D}$ in the virtual world. 3D Internet is a powerful new way for you to reach consumers, business customers, co-workers, partners, and students. It is inherently interactive and engaging. Virtual worlds provide immersive 3D experiences that reproduce real life. Despite the prevalence of 3D hardware the current set of 3D standards for the Internet have not taken off. The issues with the standards, technology, developers, end-users, or any combination of the lot as described in the paper needs to be resolved. Individuals involved in the various development uses different platforms and views need to integrate for effective delivery is highly essential.

\section{References}

Alpcan, T., Bauckhage, C., \& Kotsovinos, E. (2008). Towards 3D Internet: Why, What, and How? Deutsche Telekom Laboratories.

Correia, L. M. (2009). 3D Internet - Technologies and challenges. IST - Technical University of Lisbon, Portugal. Retrieved from http://archive.eurescom.eu/message/messageMay2009/3D Internet_Technologies_and challenges.asp

Kamel Boulos, M. N., Hetherington, L., Wheeler, S. (2007). Second Life: An overview of the potential of 3-D virtual worlds in medical and health education. Health Info. Libr. Journal, 24, 233-245. Available at http://www.ncbi.nlm.nih.gov/pubmed/18005298

Kamel Boulos, M. N., Ramloll, R., Jones, R., \& Toth-Cohen, S. (2008). Web 3D for public, environmental and occupational health: Early examples from Second Life ${ }^{\circledR}$. International Journal of Environmental Research and Public Health, 5, 290-317; DOI: 10.3390/ijerph5040290 
Kapahnke, P., Liedtke, P., Nesbigall, S., Warwas, S., \& Klusch, M. (2010). An open platform for semanticbased $3 D$ simulations in the $3 D$ Internet. German Research Center for Artificial Intelligence, Saarbrucken, Germany

Mennecke, B. E., Triplett, J. L., Hassall, L. M., Conde, Z. J., \& Heer, R. (2011). An examination of theory of embodied social presence in virtual worlds. Decision Sciences, 42(2), 413-450.

Ohnesorge, L. K. (2013). Cisco: Prepare for the 3D Internet. Triangle Business Journal. Available at http://www.bizjournals.com/triangle/blog/2013/01/cisco-prepare-for-the-3d-internet.html

Pirkola, J. (2012). 3D Internet Networked Media Systems workshop. 27th of January.

Rattner, J. (n.d.).The rise of the $3 D$ Internet. Retrieved from http://www.fi-prague.eu/program/p/rattner.pdf

Sivan, Y. (2008). 3D3C real virtual worlds defined: The immense potential of merging 3D, community, creation, and commerce. Journal of Virtual Worlds Research, 1, 1-31. Retrieved from http://journals.tdl.org/jvwr/index.php/jvwr/article/view/278

The future of the Internet. (2008). A Compendium of European Projects on ICT Research. Supported by the EU 7th Framework Programme for RTD, European commission, Information society and media.

Wunsch-Vincent, S., \& Vickery, G. (2007): Participative web: User-created content. Unclassified DSTI/ICCP/IE(2006)7/FINAL Organisation for Economic Co-operation and Development 12-Apr2007 Directorate for Science, Technology and Industry Committee for Information, Computer and Comunicatios Policy, Working Party on the Information Economy. Available at http://www.oecd.org/internet/ieconomy/38393115.pdf

Zahariadis, T., Daras, P., \& Laso-Ballesteros, I. (2009). Towards Future 3D Media Internet.

\section{Biographies}

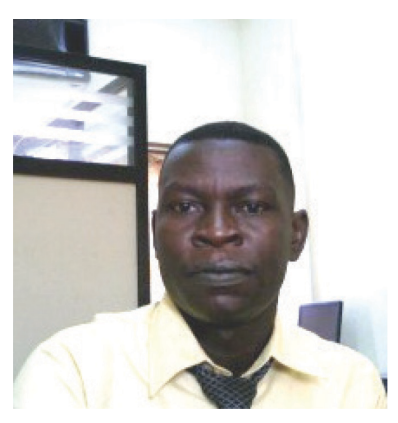

Agbaje M.O. is a lecturer and currently working on his Ph.D at Babcock University, Nigeria. His research interests are Information security, A.I and Embedded systems.

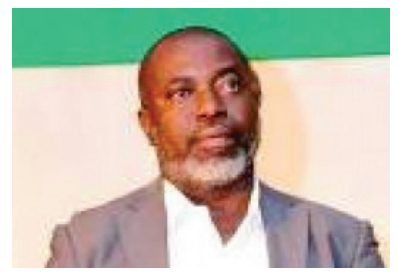

Oludele Awodele has a Ph.D in Computer Science. He is currently the H.O.D of Computer Science department, Babcock University. His areas of interest are A.I and Computer Architecture. He has published scientific articles in several journals of international repute.

Joshua J.V is a lecturer in Computer science. His areas of interest are Software engineering Computer Networking and Programming. He has published scientific articles in several journals of international repute. 